ARTICLE OPEN

\title{
Comparison of short-range order in irradiated dysprosium
} titanates

\author{
Roman Sherrod ${ }^{1}$, Eric C. O'Quinn ${ }^{1}$, Igor M. Gussev ${ }^{1}$, Cale Overstreet $\mathbb{D}^{1}$, Joerg Neuefeind $\mathbb{D}^{2}$ and Maik K. Lang (iD ${ }^{1 凶}$
}

The structural response of $\mathrm{Dy}_{2} \mathrm{TiO}_{5}$ oxide under swift heavy ion irradiation ( $2.2 \mathrm{GeV} \mathrm{Au}$ ions) was studied over a range of structural length scales utilizing neutron total scattering experiments. Refinement of diffraction data confirms that the long-range orthorhombic structure is susceptible to ion beam-induced amorphization with limited crystalline fraction remaining after irradiation to $8 \times 10^{12}$ ions $/ \mathrm{cm}^{2}$. In contrast, the local atomic arrangement, examined through pair distribution function analysis, shows only subtle changes after irradiation and is still described best by the original orthorhombic structural model. A comparison to $\mathrm{Dy}_{2} \mathrm{Ti}_{2} \mathrm{O}_{7}$ pyrochlore oxide under the same irradiation conditions reveals a different behavior: while the dysprosium titanate pyrochlore is more radiation resistant over the long-range with smaller degree of amorphization as compared to $\mathrm{Dy}_{2} \mathrm{TiO}_{5}$, the former involves more local atomic rearrangements, best described by a pyrochlore-to-weberite-type transformation. These results highlight the importance of short-range and medium-range order analysis for a comprehensive description of radiation behavior.

npj Materials Degradation (2021)5:19; https://doi.org/10.1038/s41529-021-00165-6

\section{INTRODUCTION}

Dysprosium titanate oxide $\left(\mathrm{Dy}_{2} \mathrm{TiO}_{5}\right)$ is a neutron absorbing material used in control rods in a number of nuclear reactors ${ }^{1,2}$. It is an attractive nuclear material due to dysprosium's large thermal neutron absorption cross section, as well as the low thermal expansion, large heat capacity, low swelling under irradiation, high melting point, suppression of outgassing, and chemical compatibility with cladding materials ${ }^{3,4}$. Within the extreme environment of nuclear applications, materials will be modified across multiple length-scales, from atomic-scale changes to extended long-range defective microstructures. These structural changes will affect thermomechanical and transport properties, which depend strongly on behavior at different length-scales. Therefore, as with all nuclear materials, it is imperative to understand the radiation response of $\mathrm{Dy}_{2} \mathrm{TiO}_{5}$ across length scales from the atomic arrangement to the long-range structure.

$\mathrm{Dy}_{2} \mathrm{TiO}_{5}$, like other $\mathrm{A}_{2} \mathrm{TiO}_{5} \quad\left(\mathrm{~A}=\right.$ trivalent lanthanides, $\left.\mathrm{Y}^{3+}\right)$ materials, commonly occurs in one of three types-an orthorhombic phase of space group Pnma (Fig. 1a), a hexagonal phase of space group $P 63 / \mathrm{mmc}$, and cubic phases reported as $\mathrm{Fm}-3 \mathrm{~m}, \mathrm{Fd}$ $3 m$, or $F-43 m$ depending on synthesis routes ${ }^{5}$. The orthorhombic phase is the equilibrium phase of $\mathrm{Dy}_{2} \mathrm{TiO}_{5}$ under ambient conditions and it reversibly transforms to a hexagonal hightemperature phase above $1370^{\circ} \mathrm{C}$, followed by a cubic hightemperature phase above $1680^{\circ} \mathrm{C}$, before melting at $1875^{\circ} \mathrm{C}^{5}$. In the orthorhombic phase, $\mathrm{Dy}_{2} \mathrm{TiO}_{5}$ exhibits unusual polyhedral geometries with Dy occupying 7-coordinate mono-capped octahedra and $\mathrm{Ti}$ occupying 5-coordinate square pyramids ${ }^{5}$. The radiation response of $\mathrm{A}_{2} \mathrm{BO}_{5}$ compounds is often compared and contrasted to that of compositionally-related $\mathrm{A}_{2} \mathrm{~B}_{2} \mathrm{O}_{7}$ pyrochlore materials ${ }^{6}$. These complex oxides, which are also relevant for nuclear applications, adopt either an ordered pyrochlore $(F d-3 m)$ or disordered, defect-fluorite $(F m-3 m)$ phase ${ }^{7}$. Both phases are derivatives of the ideal fluorite structure, but with two cations and one vacant anion site, which are ordered in pyrochlore and disordered in defect-fluorite ${ }^{7}$. In the ordered pyrochlore structure, the larger A-cation occupies a 8-coordinate distorted trigonal scalenohedron and the smaller B-cation occupies a 6-coordinate trigonally flattened octahedron ${ }^{8}$. The adopted structure type is determined by the ratio of cation's ionic radii $\left(r_{A} / r_{B}\right)$ as well as the cation antisite defect energy. For example, most $\mathrm{A}_{2} \mathrm{Ti}_{2} \mathrm{O}_{7}$ compounds exhibit a pyrochlore structure (e.g., $\mathrm{Dy}_{2} \mathrm{Ti}_{2} \mathrm{O}_{7}$, Fig. 1b) while some $\mathrm{A}_{2} \mathrm{Zr}_{2} \mathrm{O}_{7}$ compounds can only be synthesized with a defect-fluorite structure (e.g., $\left.\mathrm{Ho}_{2} \mathrm{Zr}_{2} \mathrm{O}_{7}\right)^{9}$.

The irradiation environment encountered by nuclear materials is harsh, complex and can be divided into two distinct regimes: (i) low energy ions (keV-MeV range), e.g., recoil nuclei from alpha-decay events, which interact with a material through ballistic collisions (nuclear energy loss regime) and (ii) high energy ions (MeV-GeV range), e.g., fragments from fission events, which interact primarily with the electrons within a material (electronic energy loss regime). $\mathrm{A}_{2} \mathrm{BO}_{5}$ compounds have been studied under both irradiation regimes and the lanthanide titanates $\left(\mathrm{Ln}_{2} \mathrm{TiO}_{5}\right)$, in general, are susceptible to radiation-induced amorphization ${ }^{6,10-13}$. Tracks with an amorphous core and a disordered shell form in $\mathrm{Ln}_{2} \mathrm{TiO}_{5}$ after $1.4 \mathrm{GeV}$ Xe ion irradiation with the amorphization efficiency increasing as a function of $\mathrm{Ln}$ cation size $\mathrm{e}^{12}$. A similar behavior is observed for low-energy ion irradiation ( $1 \mathrm{MeV} \mathrm{Kr}$ ions) of $\mathrm{Ln}_{2} \mathrm{TiO}_{5}$ by a decrease in the critical amorphization dose with increasing $\operatorname{Ln}$ cation size ${ }^{10,11}$, which is equivalent to an increase of the critical amorphization temperature, $T_{\mathrm{c}}$, above which the material can no longer be amorphized $\left(T_{\mathrm{c}}=\right.$ $400-440{ }^{\circ} \mathrm{C}$ for $\mathrm{Dy}_{2} \mathrm{TiO}_{5}{ }^{10,11}$ ).

The local order of amorphous and disordered crystalline complex oxides has recently been studied using a variety of newly developed and improved techniques ${ }^{14-20}$, including Raman spectroscopy, electron energy loss spectroscopy (EELS), extended $x$-ray absorption fine structure analysis (EXAFS), and neutron total scattering. These techniques have the advantage of providing structural information on local defects and disorder. Irradiation effects in $\mathrm{Dy}_{2} \mathrm{TiO}_{5}$ and other titanate compositions have been previously investigated using grazing incidence $x$-ray diffraction analysis $^{10}$, transmission electron microscopy $(\mathrm{TEM})^{10,11}$, and 
dilatometry $^{21}$ techniques. While these studies have provided significant insight into the process of radiation damage in dysprosium titanate, the analytical techniques used have several general shortcomings. For instance, the low interaction cross section of $x$-rays with low- $Z$ elements, such as oxygen, makes $x$-ray probes insensitive to radiation damage induced within the oxygen sublattice. In contrast, neutron probes, for which interaction cross sections have no explicit Z-dependence, provide structural information on the cation and oxygen sublattices. Neutron total scattering experiments have recently received more attention due to advent of modern, intense spallation neutron sources with beamlines specifically designed to produce high resolution pair distribution functions (PDFs), such as the Nanoscale Ordered Materials Diffractometer (NOMAD) at the Spallation Neutron Source $(\mathrm{SNS})^{22}$. The intense neutron flux of $\sim 1 \times 10^{8}$ neutrons/ $\mathrm{cm}^{2} \mathrm{~s}$ reduces the required sample mass for such experiments to $\sim 50-100 \mathrm{mg}$. This amount of irradiated sample mass is within the achievable range of the largest ion accelerator facilities ${ }^{23}$.

Previous radiation-damage studies of different pyrochlore oxides using neutron total scattering analysis have revealed local structural changes that are not represented by the average, longrange structure observed by conventional analysis. For example, Shamblin et al. showed that locally-ordered structural motifs are present in irradiated pyrochlore oxides that are arranged such that the structural information from diffraction experiments does not represent the actual local atomic configuration ${ }^{20}$. Independent of
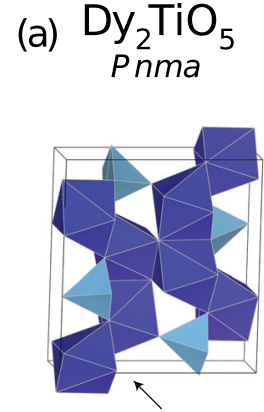

[010]
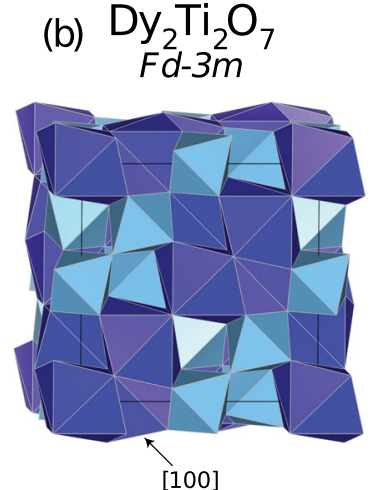

[100]
Fig. 1 3-D polyhedral renderings of the $\mathrm{Dy}_{2} \mathrm{TiO}_{5}$ and $\mathrm{Dy}_{2} \mathrm{Ti}_{2} \mathrm{O}_{7}$ starting structures. Titanium polyhedra represented by a lighter blue, and dysprosium polyhedra represented by a darker blue for a the orthorhombic, Pnma structure $\left(\mathrm{Dy}_{2} \mathrm{TiO}_{5}\right)$ and b the cubic, $F d-3 m$ pyrochlore structure $\left(\mathrm{Dy}_{2} \mathrm{Ti}_{2} \mathrm{O}_{7}\right)$. whether a pyrochlore undergoes an amorphization process during swift heavy ion irradiation (e.g., $\mathrm{Dy}_{2} \mathrm{Sn}_{2} \mathrm{O}_{7}$ ) or is disordered to the defect-fluorite structure (e.g., $\mathrm{Er}_{2} \mathrm{Sn}_{2} \mathrm{O}_{7}$ ), the short-range structure changes to a weberite-type atomic arrangement ${ }^{20}$. This suggests that radiation damage is more complex than previously thought and short-range as well as medium-range analysis with high sensitivity to the anion sublattice must be included in ion-beam studies. The local structural changes induced by ion irradiation of $\mathrm{A}_{2} \mathrm{BO}_{5}$ compounds have not been well studied and this work was performed to analyze the amorphization process of $\mathrm{Dy}_{2} \mathrm{TiO}_{5}$ across a range of length scales. Swift heavy ions were used to irradiate the material in sufficient quantity for neutron total scattering experiments. The PDFs, produced from the total scattering data, provided information on the local atomic arrangement within the aperiodic phase. The behavior was then compared with the compositionally-related $\mathrm{Dy}_{2} \mathrm{Ti}_{2} \mathrm{O}_{7}$ pyrochlore which also undergoes amorphization under ion irradiation, focusing particularly on changes to the coordination polyhedra and their connectivity.

\section{RESULTS AND DISCUSSION}

\section{Long-range structural analysis}

Neutron diffraction data of the $\mathrm{Dy}_{2} \mathrm{Ti}_{2} \mathrm{O}_{7}$ and $\mathrm{Dy}_{2} \mathrm{TiO}_{5}$ samples confirm that both starting materials are well crystallized with no discernible impurity phases (Fig. 2). The diffraction data are wellfitted by the expected structures for both samples; the data residual $\left(R_{w}\right)$ for each is below $5 \%$. After irradiation, both samples display distinct loss of long-range crystallinity evident by reduced peak intensities and strongly increased diffuse scattering (Fig. 2). This agrees well with previous synchrotron $\mathrm{x}$-ray diffraction studies which showed that $\mathrm{Ln}_{2} \mathrm{Ti}_{2} \mathrm{O}_{7}$ and $\mathrm{Ln}_{2} \mathrm{TiO}_{5}$ titanate oxides can be readily amorphized by swift heavy ions at room temperature $\mathrm{t}^{10,11,24}$. The remaining long-range crystalline phase for each irradiated sample is still well fit using the unirradiated structural model. No peak broadening or shifts are observable, indicative of undamaged pyrochlore with no significant accumulation of defects. The incomplete amorphization in this study can be explained by specifics of the ion-beam experiments. Neutron total scattering experiment requires large sample amounts which can only be achieved by irradiating large areas with a highly defocused beam spot. This may introduce inhomogeneity within the irradiated area and some sample powder may not be fully covered by the ion beam. However, both samples were irradiated under identical beam setting to $8 \times 10^{12}$ ions $/ \mathrm{cm}^{2}$ and their relative radiation behavior can be compared. Additionally, the scattering information from the unirradiated sample regions can be well separated from those arising from the modified material
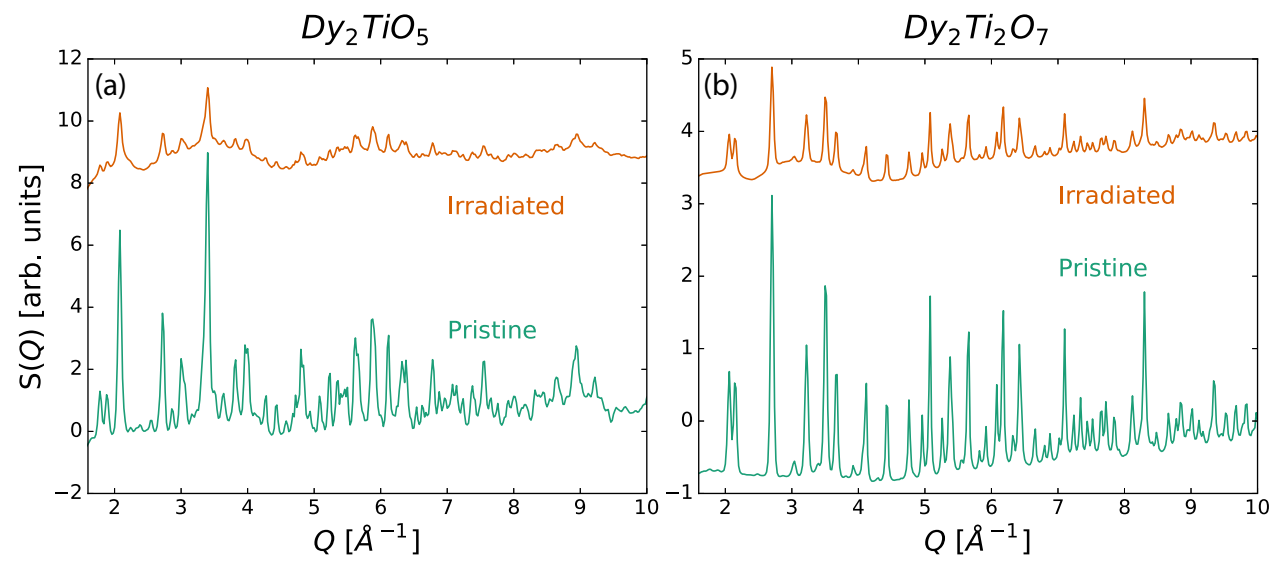

Fig. 2 Neutron total scattering structure functions of both dysprosium titanate oxides before and after ion irradiation. Diffraction pattern of (a) orthorhombic $\mathrm{Dy}_{2} \mathrm{TiO}_{5}$ and (b) cubic $\mathrm{Dy}_{2} \mathrm{Ti}_{2} \mathrm{O}_{7}$ with amorphization (loss of long-range periodicity) in both samples indicated by significant peak area reduction and increase in diffuse scattering. Irradiation was performed with $2.2 \mathrm{GeV}$ Au ions with a fluence of $8 \times 10^{12}$ ions/cm ${ }^{2}$. 
(a) $\mathrm{A}_{2} \mathrm{TiO}_{5}($ Pnma)
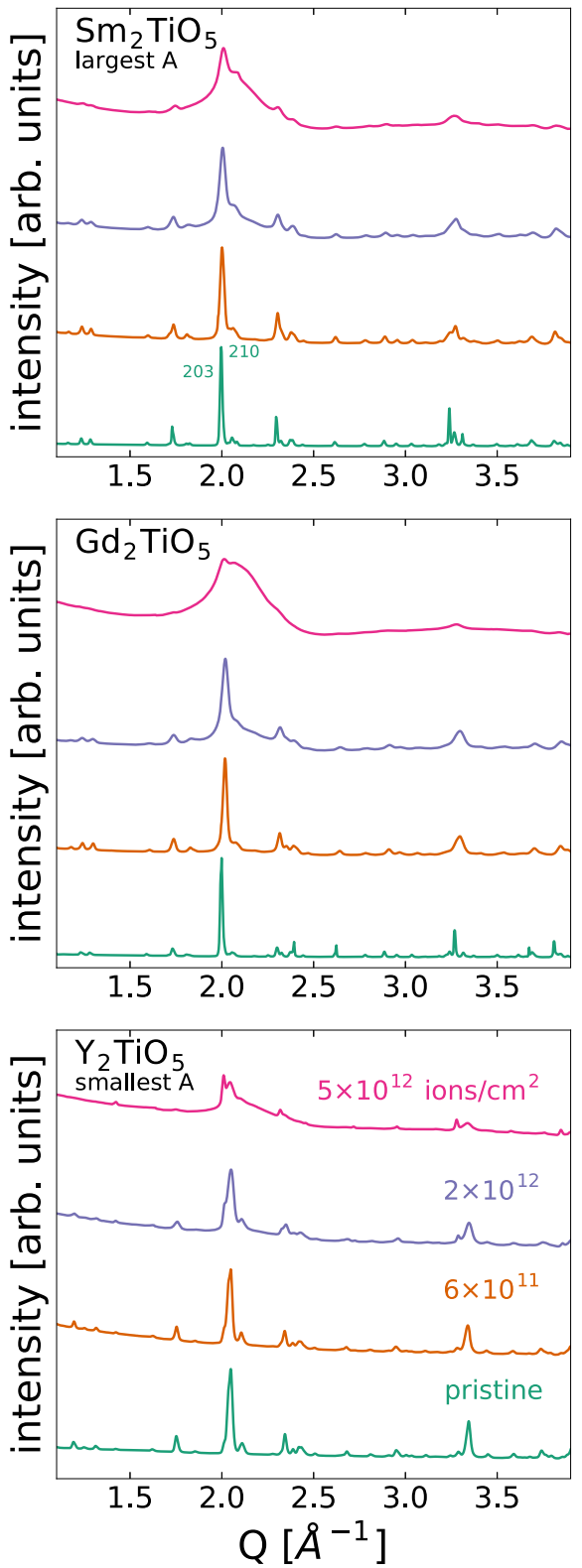

(b) $\mathrm{A}_{2} \mathrm{Ti}_{2} \mathrm{O}_{7}:(F d-3 m)$
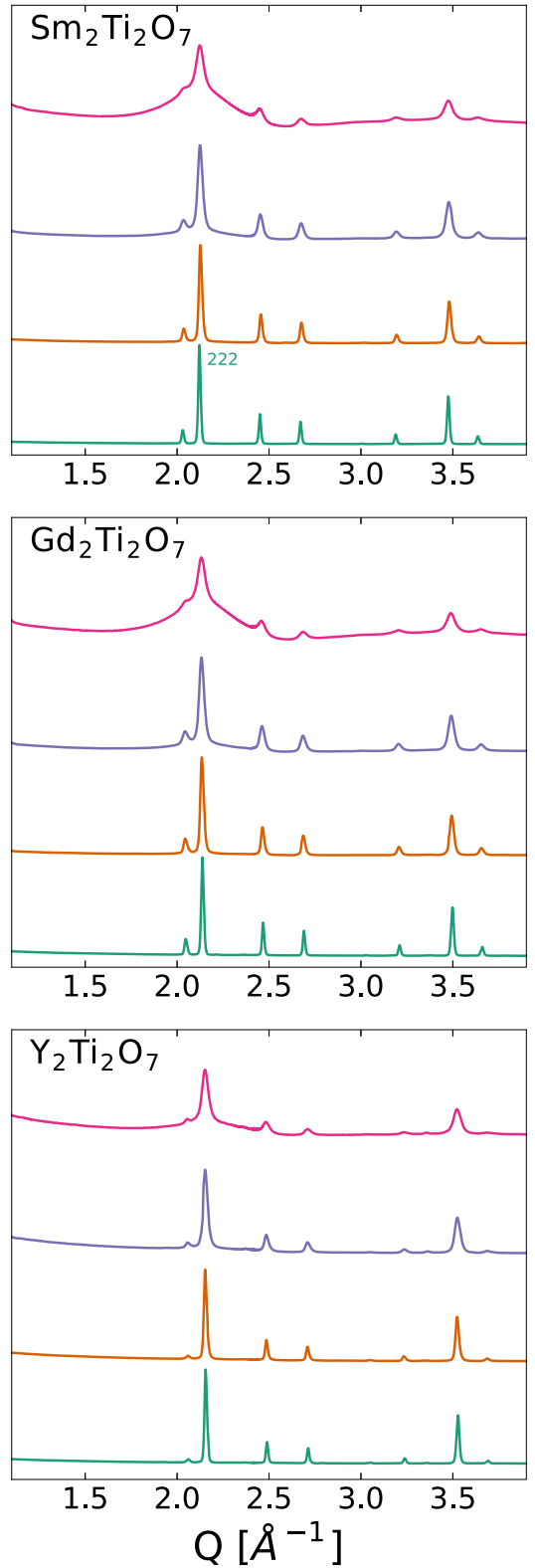

Fig. 3 Synchrotron $x$-ray diffraction patterns of titanate oxide samples before and after ion irradiation. More pronounced radiation effects are evident in diffraction patterns of $\mathbf{a}$ orthorhombic $\mathrm{A}_{2} \mathrm{TiO}_{5}$ compared to $\mathbf{b}$ cubic $\mathrm{A}_{2} \mathrm{Ti}_{2} \mathrm{O}_{7}$ with $\mathrm{A}=\mathrm{Sm}$, Gd, and $\mathrm{Y}$, particularly by the increased diffuse scattering at higher fluences. Ion fluences (in ions $/ \mathrm{cm}^{2}$ ) as indicated for $\mathrm{Y}_{2} \mathrm{TiO}_{5}$ apply to all sample compositions and irradiation was performed with $2.2 \mathrm{GeV}$ Au ions. Data are from previously published and unpublished work ${ }^{13,26,27}$.

volume. The amorphous background is significantly more pronounced in the $\mathrm{Dy}_{2} \mathrm{TiO}_{5}$ diffraction pattern with a greater relative Bragg peak-intensity reduction of the remaining crystalline phase as compared to $\mathrm{Dy}_{2} \mathrm{Ti}_{2} \mathrm{O}_{7}$. This indicates that the same swift heavy ion irradiation more efficiently amorphizes orthorhombic $\mathrm{Dy}_{2} \mathrm{TiO}_{5}$, suggesting that the cross-section for amorphization of individual ion tracks is larger than in cubic $\mathrm{Dy}_{2} \mathrm{Ti}_{2} \mathrm{O}_{7}$. This is in clear contrast to previous studies on these materials using lowenergy ions ( $1 \mathrm{MeV} \mathrm{Kr}$ ) which demonstrated that within the nuclear energy loss regime $\mathrm{A}_{2} \mathrm{TiO}_{5}$ compounds are more resistant than corresponding $\mathrm{A}_{2} \mathrm{Ti}_{2} \mathrm{O}_{7}$ compositions ${ }^{10,25}$. The long-range behavior observed for $\mathrm{Dy}_{2} \mathrm{TiO}_{5}$ and $\mathrm{Dy}_{2} \mathrm{Ti}_{2} \mathrm{O}_{7}$ under swift heavy ion irradiation seems to be a general trend across a range of chemical compositions based on previous published and unpublished data $^{13,26,27}$ (Fig. 3). Synchrotron x-ray diffraction analysis of
$\mathrm{A}_{2} \mathrm{Ti}_{2} \mathrm{O}_{7}{ }^{26}$ and $\mathrm{A}_{2} \mathrm{TiO}_{5}(\mathrm{~A}=\mathrm{Sm}, \mathrm{Gd} \text {, and } \mathrm{Y})^{13}$ irradiated with $2.2 \mathrm{GeV} \mathrm{Au}$ ions under identical conditions shows at each fluence step more pronounced radiation effects for the $\mathrm{A}_{2} \mathrm{TiO}_{5}$ materials (Fig. 3). This is particularly evident at higher fluences by the diffuse scattering band at $Q=1.5-2.5 \AA^{-1}$ produced by the amorphous phase ${ }^{15}$. These results combined with the present neutron diffraction data consistently show that, within the electronic energy loss regime of swift heavy ions, orthorhombic $\mathrm{A}_{2} \mathrm{TiO}_{5}$ compounds are more readily amorphized than the corresponding cubic $\mathrm{A}_{2} \mathrm{Ti}_{2} \mathrm{O}_{7}$ pyrochlore compositions. It remains unclear why this behavior contrasts the response under irradiation with ions of predominantly nuclear energy loss ${ }^{10,28}$. Previous studies have shown that both nuclear and electronic energy loss regimes induce very similar radiation effects in a range of pyrochlore compositions ${ }^{26}$. It should be noted that ion irradiations in the 
nuclear energy loss regime utilized mostly in situ TEM characterization on electron transparent samples (typical thickness $<100 \mathrm{~nm}$ ). The increased surface area in such nanofilms can modify damage formation and recovery as compared to the bulk samples used for this study, which may contribute to the reversed relative radiation response of $\mathrm{A}_{2} \mathrm{TiO}_{5}$ and $\mathrm{A}_{2} \mathrm{Ti}_{2} \mathrm{O}_{7}$ compounds under swift heavy ion and low-energy ion irradiation.

\section{Short-range structural analysis}

The short-range structures of the unirradiated and irradiated samples were characterized through PDF analysis. Using small-box refinement, the local and intermediate structure of pristine, unirradiated $\mathrm{Dy}_{2} \mathrm{TiO}_{5}$ from 1.5 to $20 \AA$ (Fig. 4) was well modeled with the orthorhombic structure used for Rietveld refinement of the diffraction pattern $\left(R_{w}=15 \%\right)$. Beyond the nearest-neighbor distance of $\sim 3.3 \AA$ there is a distinct reduction in peak intensity for the irradiated sample compared with the unirradiated material (Fig. 4). Notably, the peak widths, a measure of disorder, do not change significantly outside this range. Thus, the reduction in intensity is equivalent to a significant decrease in peak area consistent with a crystalline-to-amorphous transformation and associated loss of long-range coordination ${ }^{20}$. The peaks that correspond to the nearest-neighbor coordination polyhedra, however, exhibit only subtle changes after ion irradiation. This is in clear contrast to the changes observed in the PDF of irradiated $\mathrm{Dy}_{2} \mathrm{Ti}_{2} \mathrm{O}_{7}$ (Fig. 5). While the behavior beyond nearest-neighbor distances of $\sim 3 \AA$ is comparable to that of $\mathrm{Dy}_{2} \mathrm{TiO}_{5}$, with amorphization evidenced by reduction in peak area and no changes to peak width and shape, ion-beam exposure creates distinct modifications to the local atomic arrangement. The PDF of the unirradiated $\mathrm{Dy}_{2} \mathrm{Ti}_{2} \mathrm{O}_{7}$ can be best described with the $\mathrm{Fd}-3 \mathrm{~m}$ pyrochlore structural model. The PDF of the irradiated $\mathrm{Dy}_{2} \mathrm{Ti}_{2} \mathrm{O}_{7}$ remains consistent with the unirradiated pyrochlore structural model at larger $r$-values (unirradiated sample regions in agreement with diffraction data), but displays significant changes between 2 and $5 \AA$. The intensity of the negative peak at $\sim 2 \AA$ is reduced, and the distinct peaks at 2.2 and $2.5 \AA$ are replaced with a single peak at $2.3 \AA$. The peak at $3.0 \AA$ broadens and shifts to lower-r. Similar behavior has been previously reported for several irradiation-induced amorphous pyrochlore compositions ${ }^{18,20}$, and

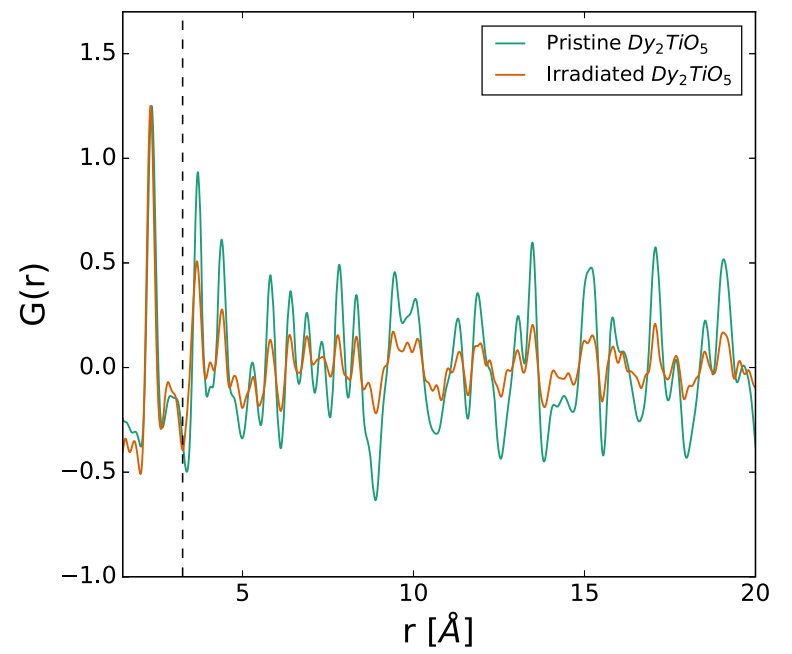

Fig. 4 Neutron pair distribution function (PDF) for pristine and irradiated $\mathrm{Dy}_{2} \mathrm{TiO}_{5} . G(r)$ is proportional to the probability of specific interatomic correlations at distance $r$. The dashed line indicates the maximum $r$-value for the coordination polyhedra region of the PDF. Loss of long-range structural coherency is evident at larger $r$-values by pronounced peak-intensity reduction with little changes to peak width. the PDF of irradiated $\mathrm{Dy}_{2} \mathrm{Ti}_{2} \mathrm{O}_{7}$ can be best modeled in a range up to $\sim 8 \AA$ with a weberite-type atomic arrangement of space group $\mathrm{Ccmm}^{20,29}$. Interestingly, the same local structural transformations are also evident in pyrochlore compositions which are radiation resistant but disorder to a defect-fluorite structure over the longrange ${ }^{19,20}$. The presence of local ordering in amorphized pyrochlores has also been confirmed using EXAFS ${ }^{14}$, Raman spectroscopy ${ }^{15}$, and EELS ${ }^{16}$. The most significant change in the PDF that occurs in irradiated $\mathrm{Dy}_{2} \mathrm{TiO}_{5}$ is a decrease in slope near $3.7 \AA$ caused primarily by a shift of the negative peak near $3.4 \AA$ (Fig. 5). Other subtle changes include a small shift of the first three positive peaks and a slight increase in intensity of the second peak near $3 \AA$. Despite its lower resistance to irradiation-induced amorphization displayed by the Bragg peak response of the long-range structure, $\mathrm{Dy}_{2} \mathrm{TiO}_{5}$ has much less structural modifications at the level of the coordination polyhedra than $\mathrm{Dy}_{2} \mathrm{Ti}_{2} \mathrm{O}_{7}$.

To investigate the subtle changes of the local structure in $\mathrm{Dy}_{2} \mathrm{TiO}_{5}$ before and after irradiation in more detail, the PDF of the irradiated sample was modeled with the initial orthorhombic, Pnma structure. This structural model describes well the PDF between 1.5 and $8 \AA$ (Fig. $6, R_{w}=16 \%$ ), despite minor deviations in peak shape and position. The spatial extent of structural changes to the local structure in $\mathrm{Dy}_{2} \mathrm{TiO}_{5}$ is similar to the length scale observed by Shamblin et al. ${ }^{20}$ and Chung et al. ${ }^{18}$ for radiation-induced weberite-type ordering in amorphized $\mathrm{Dy}_{2} \mathrm{Sn}_{2} \mathrm{O}_{7}$ and $\mathrm{Dy}_{2} \mathrm{Ti}_{2} \mathrm{O}_{7}$, respectively. To gain further insight into these local structural changes, induced by the swift heavy ion irradiation of $\mathrm{Dy}_{2} \mathrm{TiO}_{5}$, the partial PDF contributions from each atom-atom pair in the corresponding refinements were calculated (individual peaks positions are indicated by arrows in Fig. 6). The region with the most pronounced changes in the PDF profile ( $r$-values between 3 and $4 \AA$ ) is a superposition of contributions from 4 distinct atom-atom pairs, making it difficult to unambiguously discern the origin of the observed subtle changes in the PDF before and after ion irradiation. The oxygen-oxygen pair correlations at $\sim 3.0 \AA$ and the Dy-Ti nearest-neighbor correlation at $\sim 3.5 \AA$ appear to have the most noticeable changes. These observations combined with the Dy-O and Ti-O nearest-neighbor correlations remaining mostly unchanged after irradiation, suggest that long-range amorphization in $\mathrm{Dy}_{2} \mathrm{TiO}_{5}$ is locally

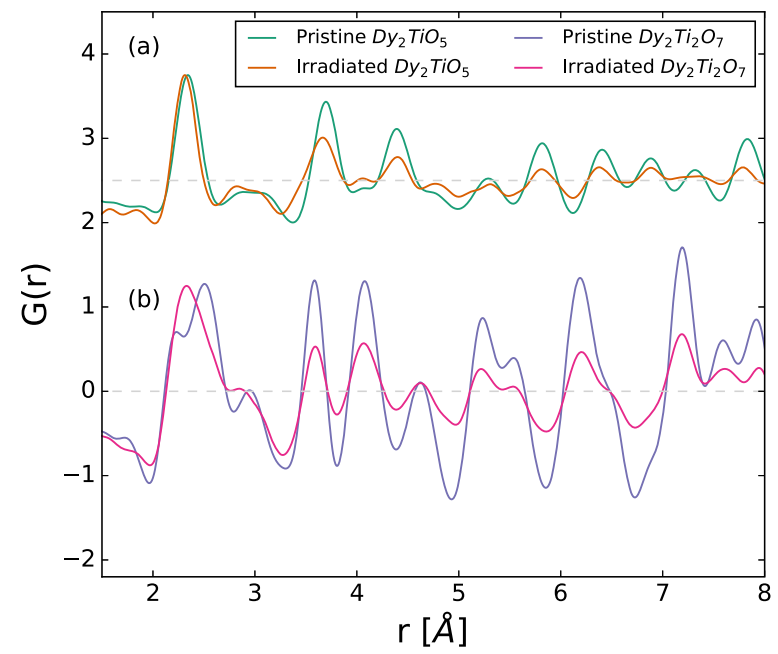

Fig. 5 Neutron pair distribution function (PDF) for pristine and irradiated $\mathrm{Dy}_{2} \mathrm{TiO}_{5}$ and $\mathrm{Dy}_{2} \mathrm{Ti}_{2} \mathrm{O}_{7}$. a Only subtle changes are evident in the PDF of $\mathrm{Dy}_{2} \mathrm{TiO}_{5}$ after ion irradiation, most notable at $\sim 3.5 \AA$ corresponding to the negative Dy-Ti correlation and the positive Dy-Dy/O-O correlations. b The PDF of pyrochlore-structured $\mathrm{Dy}_{2} \mathrm{Ti}_{2} \mathrm{O}_{7}$ instead shows significant changes that are consistent with an atomic rearrangement to a weberite-type phase. 


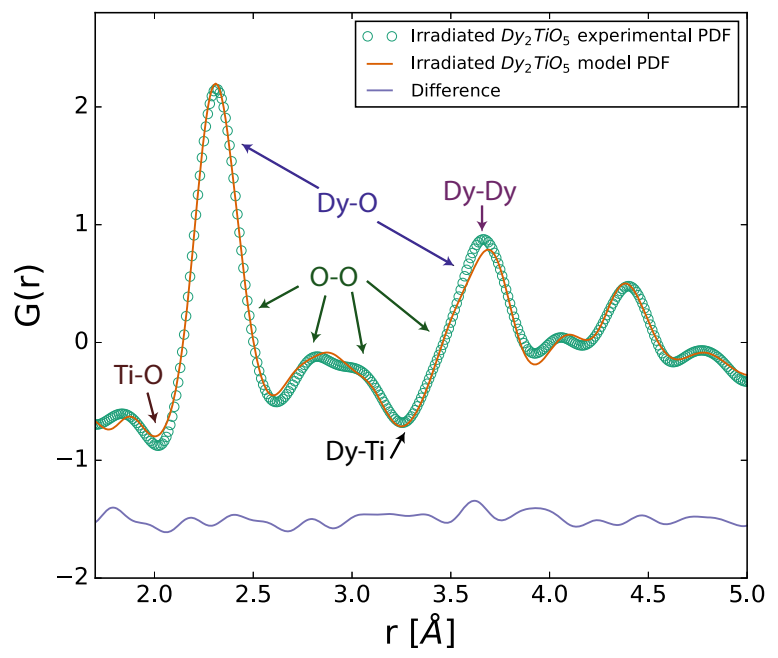

Fig. 6 Neutron PDF for irradiated $\mathrm{Dy}_{2} \mathrm{TiO}_{5}$ with small-box refinement. The refinement based on the pristine orthorhombic structural model (orange line) describes the experimental data (green circles) well as indicated by the flat difference curve (purple line). The major atomic correlations corresponding to peaks in the PDF for $\mathrm{Dy}_{2} \mathrm{TiO}_{5}$ are labeled based on the applied structural model.

accompanied by slight distortions of the coordination polyhedra and modification of the connectivity between these structural building blocks. Nevertheless, the polyhedra stay intact and are resistant to irradiation which explains why locally the orthorhombic structural model still describes the atomic arrangement. The short-range structures in the irradiated and unirradiated samples are two variants of the same structural model. This is in clear contrast to the formation of a weberite-type atomic arrangement in irradiated pyrochlore, which requires distinct changes to the coordination polyhedra. In the fully ordered $\mathrm{Dy}_{2} \mathrm{Ti}_{2} \mathrm{O}_{7}$ pyrochlore structure, Dy is in eightfold coordination and shares multiple polyhedral edges, while $\mathrm{Ti}$ is in sixfold coordination and shares all corners (Fig. 1b). The weberite-type arrangement induced by ion irradiation contains similar eightfold coordinated edge sharing Dy and sixfold coordinated corner sharing $\mathrm{Ti}$, but also has sevenfold coordinated edge sharing mixed cation sites. The orthorhombic $\mathrm{Dy}_{2} \mathrm{TiO}_{5}$ structure has sevenfold coordinated, edge sharing Dy polyhedra and fivefold coordinated Ti-polyhedra sharing 2 corners (Fig. 1a). The smaller number of shared components in the Tipolyhedra of $\mathrm{Dy}_{2} \mathrm{TiO}_{5}$ may result in greater structural stability. Further, the Ti-polyhedra in $\mathrm{Dy}_{2} \mathrm{TiO}_{5}$ have a lower coordination (5-coordinated) as compared to Ti-polyhedra in $\mathrm{Dy}_{2} \mathrm{Ti}_{2} \mathrm{O}_{7}$ (6coordinated), which results in stronger bonds in the former as the excess $\mathrm{Ti}$ charge is shared between a smaller number of oxygen atoms. This greater bond strength within the coordination polyhedra with the reduced number of sharing elements may be a key component to the improved radiation stability across the short-range structure in $\mathrm{Dy}_{2} \mathrm{TiO}_{5}{ }^{30,31}$. Why this behavior does not translate also into an enhanced amorphization resistance over the long-range may be related to the intermediate-range structure and the ability to stack local atomic building blocks to maintain long-range coherency.

Swift heavy ions induce highly transient, nonequilibrium conditions in a material that result in energy densities far exceeding binding energies within track regions. Complex damage morphologies are often quenched in complex oxides such as pyrochlore consisting of an amorphous core and a disordered shell ${ }^{12,26,30}$. The present neutron data comprise the entire modified material volume created by overlapping ion tracks and represent the overall structural behavior after quenching and defect recovery. It remains unclear whether the coordination polyhedra adopt the observed configurations during the transient conditions or quenching, but the resulting atomic arrangement is dictated by a set of fundamental chemical rules. The nature of local order in disordered complex oxides was recently described by O'Quinn et al. ${ }^{32}$ based on an extension of Pauling's rules ${ }^{33}$. While disorder from irradiation was not included in this previous study, a similar approach may also be applicable to explain the different atomic rearrangements in both dysprosium titanate oxides that are induced by swift heavy ion irradiation. The reduced number of sharing elements in the coordination polyhedra of $\mathrm{Dy}_{2} \mathrm{TiO}_{5}$ coupled with the stoichiometry and chemistry of the atomic constituents provide reduced structural flexibility and the final atomic configurations must reassemble the original coordination polyhedra. In contrast, greater structural flexibility in $\mathrm{Dy}_{2} \mathrm{Ti}_{2} \mathrm{O}_{7}$ coordination polyhedra gives access to a less constrained weberite-type atomic arrangement that is more readily accessible under highly transient extreme conditions. It remains unclear why a larger degree of amorphization is observed for the compound that largely maintains the local coordination polyhedra. Structural rigidity in $\mathrm{Dy}_{2} \mathrm{TiO}_{5}$ may also limit the ability of reconnecting individual atomic building blocks to maintain long-range coherency after disruption by the highly transient irradiation regime. Thus, changes to the intermediate-range structure induced by the ion irradiation must play an important role in the radiation response of complex oxides. This is supported by a previous study ${ }^{20}$ confirming the same weberite-type atomic arrangement in irradiated pyrochlore whether or not the long-range structure is amorphous (e.g., $\mathrm{Dy}_{2} \mathrm{Sn}_{2} \mathrm{O}_{7}$ ) or crystalline but disordered (e.g., $\mathrm{Er}_{2} \mathrm{Sn}_{2} \mathrm{O}_{7}$ ). Further studies are needed to systematically characterize the atomicscale behavior of other complex oxides under swift heavy ion irradiation with the aim to identify the structural pathway and role of medium-range structure in the amorphization process.

The ion-induced atomic-scale changes in both amorphizable materials appear to be subtle in their appearance, but they are indicative of two distinct amorphization processes. While ioninduced modifications occur over picosecond timeframes and neutron measurements are only performed on the fully quenched damaged structure, the present results lead to the following conclusions with regard to amorphization in $\mathrm{Dy}_{2} \mathrm{Ti}_{2} \mathrm{O}_{7}$ : (i) atomic bonds are disrupted and reconnected to form weberite-type coordination polyhedra and (ii) these weberitetype building blocks are randomized across the intermediaterange structure resulting in the loss of long-range coherency. In contrast, (i) appears to be different in $\mathrm{Dy}_{2} \mathrm{TiO}_{5}$ with only subtle changes to the coordination polyhedra after bond disruption and reconnection. The higher rate of amorphization in this material must be related to a very efficient randomization the same structural building blocks over the intermediate-range structure. This indicates that ion-induced modifications at the intermediate-range structure play an important role in longrange amorphization by rearrangement of local structural building blocks and the associated loss of their connectivity. Under intense ion irradiation, $\mathrm{Dy}_{2} \mathrm{Ti}_{2} \mathrm{O}_{7}$ will maintain its longrange structure to much higher ion fluences than $\mathrm{Dy}_{2} \mathrm{TiO}_{5}$ until it becomes fully amorphous (Figs. 2 and 3). However, the latter performs much better in terms of maintaining the local atomic bonding environment (Fig. 5). The local transformation to weberite-type domains in $\mathrm{Dy}_{2} \mathrm{Ti}_{2} \mathrm{O}_{7}$ with coexisting pyrochlore domains involve a larger structural mismatch as compared with $\mathrm{Dy}_{2} \mathrm{TiO}_{5}$ which retains a much more homogenous structural environment during build-up of amorphization. If the same structural behavior is present under irradiation within the nuclear energy loss regime remains an open question. The use of $\mathrm{Dy}_{2} \mathrm{TiO}_{5}$ as control rod material entails intense neutron irradiation and damage that is more closely related to $1 \mathrm{MeV} \mathrm{Kr}$ ion irradiation ${ }^{25}$. The $2.2 \mathrm{GeV}$ Au ion irradiation was chosen in 
this study to reach the large sample mass required for neutron characterization. Similar studies should be completed on these materials after irradiation within a nuclear reactor which would also provide access to a sufficiently large amount of irradiated bulk material for neutron characterization. Such experiments would reveal the atomic-scale processes related to amorphization induced in the nuclear energy loss regime, shedding light on the question why $\mathrm{A}_{2} \mathrm{TiO}_{5}$ materials are more resistant to amorphization under low energy ion irradiation, while $\mathrm{A}_{2} \mathrm{Ti}_{2} \mathrm{O}_{7}$ compounds have a greater resistance when exposed to swift heavy ions.

\section{METHODS}

\section{Sample preparation and ion-beam experiments}

Polycrystalline dysprosium titanate oxides $\left(\mathrm{Dy}_{2} \mathrm{TiO}_{5}\right.$ and $\left.\mathrm{Dy}_{2} \mathrm{Ti}_{2} \mathrm{O}_{7}\right)$ were prepared with conventional solid-state methods. Stoichiometric quantities of dysprosium sesquioxide $\left(\mathrm{Dy}_{2} \mathrm{O}_{3}\right)$ and titanium dioxide $\left(\mathrm{TiO}_{2}\right)$ powders were obtained from Alpha Aesar (99.9\% purity) and mixed in acetone. These mixtures were then formed into pellets with a uniaxial hydraulic press. The as-pressed pellets were sintered at $1200^{\circ} \mathrm{C}$ for $20 \mathrm{~h}$, ground into powder, repressed, and heated at $1400^{\circ} \mathrm{C}$ for an additional $20 \mathrm{~h}$. The resulting material was subsequently reground into polycrystalline powders for use in ion-beam irradiation and neutron characterization experiments. Laboratory $\mathrm{x}$-ray diffraction was utilized for quality control after synthesis and the obtained starting materials were pure orthorhombic $\left(\mathrm{A}_{2} \mathrm{TiO}_{5}\right)$ and cubic $\left(\mathrm{A}_{2} \mathrm{Ti}_{2} \mathrm{O}_{7}\right)$ structures, respectively.

Powders of $\mathrm{Dy}_{2} \mathrm{Ti}_{2} \mathrm{O}_{7}$ and $\mathrm{Dy}_{2} \mathrm{TiO}_{5}$ were pressed into milled cylindrical depressions in aluminum platelets with diameters of $10 \mathrm{~mm}$ and depths of $50 \mu \mathrm{m}$. Six of these platelets per sample composition were irradiated simultaneously to a fluence of $8 \times 10^{12}$ ions $/ \mathrm{cm}^{2}$, with a flux of $1 \times 10^{9}$ ions $/ \mathrm{cm}^{2} \mathrm{~s}$ chosen to minimize sample heating. Irradiation was performed at room temperature with $2.2 \mathrm{GeV} \mathrm{Au}$ ion projectiles and a $5 \times 5 \mathrm{~cm}^{2}$ beam spot. The sample mass per holder was determined according to SRIM calculations ${ }^{34}$ to ensure homogeneous energy deposition throughout the sample (ions completely penetrated the sample platelets). Because the ion energy was in the GeV range, the nuclear energy loss was negligible in comparison to the electronic energy loss (about three orders of magnitude lower). The electronic energy loss per unit path length $(\mathrm{d} E / \mathrm{d} x)$ was nearly identical for both materials with $42 \pm 2 \mathrm{keV} / \mathrm{nm}$ for $\mathrm{Dy}_{2} \mathrm{TiO}_{5}$ and $43 \pm 3 \mathrm{keV} / \mathrm{nm}$ for $\mathrm{Dy}_{2} \mathrm{Ti}_{2} \mathrm{O}_{7}$, assuming a theoretical density of $6.94 \mathrm{~g} / \mathrm{cm}^{3}$ for $\mathrm{Dy}_{2} \mathrm{TiO}_{5}$ and $6.87 \mathrm{~g} / \mathrm{cm}^{3}$ for $\mathrm{Dy}_{2} \mathrm{Ti}_{2} \mathrm{O}_{7}$ $(\mathrm{d} E / \mathrm{d} x$ given as average value \pm maximum deviation within the chosen sample depth). The sample thickness was limited to ensure full penetration of swift heavy ions utilizing ion-range calculations based on the SRIM code applying a density correction factor. The density of the powder samples was estimated to be $60 \%$ theoretical density, a value that has been shown to be an upper limit obtained by uniaxial powder compression at room temperature ${ }^{35}$. More detailed information regarding the sample preparation and irradiation procedure is provided elsewhere ${ }^{20,36}$. After ion irradiation, the pressed sample compacts were removed from the Al holders with a dull needle and were reground into fine powder with an agate mortar and pestle.

\section{Neutron total scattering experiments and structural analysis}

Neutron total scattering experiments were performed on the irradiated and pristine powders at the NOMAD instrument, Spallation Neutron Source (SNS), Oak Ridge National Laboratory (ORNL) ${ }^{22}$. A total of $\sim 75 \mathrm{mg}$ of each powder was utilized for the neutron measurements using $2 \mathrm{~mm}$ diameter quartz capillaries $(0.01 \mathrm{~mm}$ wall thickness) as sample containment. Measurements were performed at room temperature for $2 \mathrm{~h}$ and the ${ }^{3} \mathrm{He}$ detectors were calibrated using scattering data from silicon and diamond powder. The scattering data were corrected for multiple scattering and absorption ${ }^{37}$ and background correction was obtained by subtracting the scattering from an empty $2 \mathrm{~mm}$ reference quartz capillary. Details of this process are described elsewhere ${ }^{17}$. The structure function, $S(Q)$, was created by normalizing the corrected intensity to the scattering intensity from vanadium. The PDF, $G(r)$, was created with STOG $^{38}$ using a Fourier transformation of $S(Q)$ :

$G(r)=\frac{2}{\pi} \int_{Q_{\min }}^{Q_{\max }} Q[S(Q)-1] \sin [Q r] d Q$ where $Q_{\min }$ and $Q_{\max }$ equaled 0.5 and $26.0 \AA^{-1}$, respectively, for the measurements performed in this study. $Q$ is defined by:

$Q=\frac{4 \pi \sin (\theta)}{\lambda}$

where $\theta$ is the scattering angle and $\lambda$ is the neutron wavelength.

The average long-range structure of the samples was examined using Rietveld refinement of the diffraction data employing the GSAS-II software package ${ }^{39-41}$. The expected $\mathrm{Dy}_{2} \mathrm{TiO}_{5}$ orthorhombic structure (space group 62, Pnma) and the expected $\mathrm{Dy}_{2} \mathrm{Ti}_{2} \mathrm{O}_{7}$ pyrochlore structure (space group 227, $F d-3 m$ ) for the unirradiated reference materials were obtained from the Inorganic Crystal Structure Database (ICSD $)^{42-44}$. Refinement was performed using the diffraction data from detector banks 3 and 5 of the 6 banks at the NOMAD instrument which provided a balance of $Q$-range and resolution. Sequential structural refinements were performed using a 6 factor Chebyshev background curve, a scale factor, lattice parameters, lattice positions, isotropic thermal displacement parameters subject to the symmetry constraints of the chosen model, and a strain term. Initially, the pristine sample data were refined; then, the refined structure was used as a starting point for the refinement of the irradiated sample data.

The local structure of the samples was analyzed through real-space smallbox refinement using the PDFgui program ${ }^{45}$ by fitting a structure model to the obtained PDF. The PDF describes the relative probability of interatomic distances compared to that expected for uniformly distributed atoms. Therefore, a positive peak at a distance, $r$, in the PDF arise from pairs of atoms $r$ distance apart. However, titanium nuclei possess a negative neutron scattering length, and thus the $\mathrm{Dy}-\mathrm{Ti}$ and $\mathrm{O}-\mathrm{Ti}$ pair correlations manifest as negative peaks in the neutron $\mathrm{PDF}^{46}$. The $\mathrm{Dy}_{2} \mathrm{TiO}_{5}$ small-box refinements were performed through sequential fits of a scale factor, lattice parameters, lattice position variables, a single isotropic thermal displacement parameter for each element, and an atomic correlation factor. To approximate the amorphous phases, the PDF of the irradiated samples were also refined with the "spdiameter" parameter which corresponds to the spatial extent of the nanoparticle. The $\mathrm{Dy}_{2} \mathrm{Ti}_{2} \mathrm{O}_{7}$ refinements were performed using a scale factor, lattice parameters, lattice position variables, all symmetry-allowed distinct isotropic and anisotropic thermal parameters, and an atomic correlation factor ${ }^{18}$. The starting structural configuration for each data set was taken from the GSAS-II refinement of the respective longrange structure. For neutron PDF data of ion irradiated samples, alternate phases were investigated as potential models for the post-irradiation local structures. These included a weberite-type phase (space group $(\mathrm{cmm}$ ), as well as some other phases related to the chemical composition of the material (e.g., the cubic F-43m structure for $\mathrm{Dy}_{2} \mathrm{TiO}_{5}$ ).

\section{DATA AVAILABILITY}

The authors declare that all other data supporting the findings of this study are available within the paper. Raw data were generated at the Spallation Neutron Source large-scale facility. Derived data supporting the findings of this study are available from the corresponding author upon request.

Received: 2 December 2020; Accepted: 18 March 2021; Published online: 16 April 2021

\section{REFERENCES}

1. Sinha, A. \& Sharma, B. P. Development of dysprosium titanate based ceramics. J. Am. Ceram. Soc. 88, 1064-1066 (2005).

2. Risovany, V. D., Varlashova, E. E. \& Suslov, D. N. Dysprosium titanate as an absorber material for control rods. J. Nucl. Mater. (2000) https://doi.org/10.1016/ S0022-3115(00)00129-X.

3. Panneerselvam, G. et al. Thermophysical measurements on dysprosium and gadolinium titanates. J. Nucl. Mater. 327, 220-225 (2004).

4. Lee, B.-H., Kim, H.-S., Lee, S.-H. \& Sohn, D.-S. Measurement of the thermal properties of gadolinium and dysprosium titanate. Thermochim. Acta 455, 100-104 (2007).

5. Shepelev, Y. F. \& Petrova, M. A. Crystal structures of $\mathrm{Ln}_{2} \mathrm{TiO}_{5}(\mathrm{Ln}=\mathrm{Gd}, \mathrm{Dy})$ polymorphs. Inorg. Mater. 44, 1354-1361 (2008).

6. Yang, D. Y. et al. Structure and radiation effect of Er-stuffed pyrochlore $\mathrm{Er}_{2}\left(\mathrm{Ti}_{2}\right.$ $\left.{ }_{-\mathrm{x}} \mathrm{Er}_{\mathrm{x}}\right) \mathrm{O}_{7-\mathrm{x} / 2}(\mathrm{x}=0-0.667)$. Nucl. Instrum. Methods Phys. Res. Sect. B Beam Interact. Mater. At. 356-357, 69-74 (2015).

7. Subramanian, M. A., Aravamudan, G. \& Subba Rao, G. V. Oxide pyrochlores-a review. Prog. Solid State Chem. 15, 55-143 (1983). 
8. Ewing, R. C., Weber, W. J. \& Lian, J. Nuclear waste disposal-pyrochlore $\left(A_{2} B_{2} O_{7}\right)$ : nuclear waste form for the immobilization of plutonium and 'minor' actinides. J. Appl. Phys. 95, 5949-5971 (2004).

9. Sickafus, K. E. et al. Radiation tolerance of complex oxides. Science 289, 748-751 (2000).

10. Aughterson, R. D. et al. Ion-irradiation resistance of the orthorhombic $\mathrm{Ln}_{2} \mathrm{TiO}_{5}$ ( $\mathrm{Ln}=\mathrm{La}, \mathrm{Pr}, \mathrm{Nd}, \mathrm{Sm}, \mathrm{Eu}, \mathrm{Gd}, \mathrm{Tb}$ and Dy) series. J. Nucl. Mater. 467, 683-691 (2015).

11. Zhang, F. et al. lon-irradiation-induced structural transitions in orthorhombic $\mathrm{Ln}_{2} \mathrm{TiO}_{5}$. Acta Mater. 61, 4191-4199 (2013).

12. Tracy, C. L. et al. Structural response of $A_{2} T_{i O}(A=L a, N d, S m, G d)$ to swift heavy ion irradiation. Acta Mater. 60, 4477-4486 (2012)

13. Park, $S$. et al. Swift-heavy ion irradiation response and annealing behavior of $\mathrm{A}_{2} \mathrm{TiO}_{5}(\mathrm{~A}=\mathrm{Nd}, \mathrm{Gd}$, and $\mathrm{Yb})$. J. Solid State Chem. 258, 108-116 (2018).

14. Sattonnay, $G$. et al. Key role of the short-range order on the response of the titanate pyrochlore $\mathrm{Y}_{2} \mathrm{Ti}_{2} \mathrm{O}_{7}$ to irradiation. Phys. Rev. B 94, 224109 (2016).

15. Tracy, C. L. et al. Role of composition, bond covalency, and short-range order in the disordering of stannate pyrochlores by swift heavy ion irradiation. Phys. Rev. B Condens. Matter Mater. Phys 94, 1-11 (2016).

16. Sachan, R. et al. Forging fast ion conducting nanochannels with swift heavy ions: the correlated role of local electronic and atomic structure. J. Phys. Chem. C. 121, 975-981 (2017).

17. O'Quinn, E. C. et al. Advanced characterization technique for mechanochemically synthesized materials: neutron total scattering analysis. J. Mater. Sci. 53, 13400-13410 (2018).

18. Chung, C. K. et al. Thermodynamic and structural evolution of $\mathrm{Dy}_{2} \mathrm{Ti}_{2} \mathrm{O}_{7}$ pyrochlore after swift heavy ion irradiation. Acta Mater. 145, 227-234 (2018).

19. Shamblin, J. et al. Probing disorder in isometric pyrochlore and related complex oxides. Nat. Mater. 15, 507 (2016).

20. Shamblin, J. et al. Similar local order in disordered fluorite and aperiodic pyrochlore structures. Acta Mater. 144, 60-67 (2018).

21. Kosenkov, V. M., Guseva, T. M., Alekseeva, S. A. \& Nevorotin, V. K. Structural changes in irradiated dysprosium titanate. Sov. Energy 40, 513-515 (1976).

22. Neuefeind, J., Feygenson, M., Carruth, J., Hoffmann, R. \& Chipley, K. K. The nanoscale ordered materials diffractometer NOMAD at the spallation neutron source SNS. Nucl. Instrum. Methods Phys. Res. Sect. B-Beam Interact. Mater. At. 287, 68-75 (2012).

23. Lang, M., O'Quinn, E., Neuefeind, J. \& Trautmann, C. Characterization of radiation effects and ion tracks with spallation neutron probes. Nucl. Phys. N. 30, 16-19 (2020).

24. Yang, D. et al. Insights into the radiation behavior of $\mathrm{Ln}_{2} \mathrm{TiO}_{5}(\mathrm{Ln}=\mathrm{La}-\mathrm{Y})$ from defect energetics. Comput. Mater. Sci. 139, 295-300 (2017).

25. Aughterson, R. D., Zaluzec, N. J. \& Lumpkin, G. R. Synthesis and ion-irradiation tolerance of the $\mathrm{Dy}_{2} \mathrm{TiO}_{5}$ polymorphs. Acta Mater. 204, 116518 (2020).

26. Shamblin, J. et al. Structural response of titanate pyrochlores to swift heavy ion irradiation. Acta Mater. 117, 207-215 (2016).

27. Lang, M. et al. unpublished data.

28. Lian, J. et al. Radiation-Induced amorphization of rare-Earth titanate pyrochlores. Phys. Rev. B - Condens. Matter Mater. Phys. 68, 1-9 (2003).

29. Cai, L. \& Nino, J. C. Complex ceramic structures. I. Weberites. Acta Crystallogr. Sect. B Struct. Sci. 65, 269-290 (2009).

30. Park, S. et al. $\mathrm{A}_{2} \mathrm{TiO}_{5}(\mathrm{~A}=\mathrm{Dy}, \mathrm{Gd}, \mathrm{Er}, \mathrm{Yb})$ at High Pressure. Inorg. Chem. 57, 2269-2277 (2018).

31. Brown, I. D. Bond valences-a simple structural model for inorganic chemistry. Chem. Soc. Rev. 7, 359-376 (1978).

32. O'Quinn, E. C. et al. Predicting short-range order and correlated phenomena in disordered crystalline materials. Sci. Adv. 6, 1-8 (2020).

33. Pauling, L. The principles determining the structure of complex ionic crystals. J. Am. Chem. Soc. 51, 1010-1026 (1929).

34. Ziegler, J. F., Ziegler, M. D. \& Biersack, J. P. SRIM-The stopping and range of ions in matter (2010). Nucl. Instrum. Methods Phys. Res. Sect. B-Beam Interact. Mater. At. 268, 1818-1823 (2010)

35. Lang, M. et al. Swift heavy ion-induced phase transformation in $\mathrm{Gd}_{2} \mathrm{O}_{3}$. Nucl. Instrum. Methods Phys. Res. Sect. B Beam Interact. Mater. At. 326, 121-125 (2014).

36. Palomares, R. I. et al. Defect accumulation in swift heavy ion-irradiated $\mathrm{CeO}_{2}$ and $\mathrm{ThO}_{2}$. J. Mater. Chem. A 5, 12193-12201 (2017).

37. Rouse, K. D., Cooper, M. J., York, E. J. \& Chakera, A. Absorption corrections for neutron diffraction. Acta Crystallogr. Sect. a-Cryst. Phys. Diffr. Theor. Gen. Crystallogr. A 26, 682- (1970).

38. Tucker, M. G., Keen, D. A., Dove, M. T., Goodwin, A. L. \& Hui, Q. RMCProfile: reverse Monte Carlo for polycrystalline materials. J. Phys.-Condens. Matter 19, 16 (2007).

39. von Dreele, R. B. General structure analysis system (GSAS). Rep. LAUR 86-748 1-224 (2000)

40. Toby, B. H. \& Von Dreele, R. B. What's new in GSAS-II. Powder Diffr. 29, S2-S6 (2014).

41. Toby, B. H. \& Von Dreele, R. B. GSAS-Il: the genesis of a modern open-source all purpose crystallography software package. J. Appl. Crystallogr. 46, 544-549 (2013).
42. Belsky, A., Hellenbrandt, M., Karen, V. L. \& Luksch, P. New developments in the Inorganic Crystal Structure Database (ICSD): accessibility in support of materials research and design. Acta Crystallogr. Sect. B-Struct. Sci. 58, 364-369 (2002).

43. Fluck, E. Inorganic crystal structure database (ICSD) and standardized data and crystal chemical characterization of inorganic structure types (TYPIX) - Two tools for inorganic chemists and crystallographers. J. Res. Natl Inst. Stand. Technol. 101, 217-220 (1996).

44. Aughterson, R. D. et al. Crystal chemistry of the orthorhombic $\mathrm{Ln}_{2} \mathrm{TiO}_{5} \mathrm{com}$ pounds with $\mathrm{Ln}=\mathrm{La}, \mathrm{Pr}, \mathrm{Nd}, \mathrm{Sm}, \mathrm{Gd}$, Tb and Dy. J. Solid State Chem. 227, 60-67 (2015).

45. Farrow, C. L. et al. PDFfit2 and PDFgui: computer programs for studying nanostructure in crystals. J. Phys. Condens. Matter 19, 335219 (2007).

46. Sears, V. F. Neutron scattering lengths and cross sections. Neutron N. 3, 26-37 (1992).

\section{ACKNOWLEDGEMENTS}

This work was supported by the U.S. Department of Energy, Office of Science, Basic Energy Sciences, under Award DE-SC0020321. The research at ORNL's Spallation Neutron Source was sponsored by the Scientific User Facilities Division, Office of Basic Energy Sciences, U.S. Department of Energy. I.M.G. acknowledges support from the U.S. Department of Energy, Office of Science, Office of Workforce Development for Teachers and Scientists, Office of Science Graduate Student Research (SCGSR) program. The SCGSR program is administered by the Oak Ridge Institute for Science and Education (ORISE) for the DOE. ORISE is managed by ORAU under contract number DE-SC0014664. All opinions expressed in this paper are the author's and do not necessarily reflect the policies and views of DOE, ORAU, or ORISE. C.O acknowledges support from the Center for Materials Processing (CMP) at the University of Tennessee, Knoxville. The authors gratefully acknowledge technical support during the neutron scattering experiments by Michelle Everett (Oak Ridge National Laboratory) and editing support by Devon Drey (University of Tennessee). The authors wish to express their thanks to anonymous reviewers for constructive remarks which improved the manuscript.

\section{AUTHOR CONTRIBUTIONS}

R.S., E.C.O., and M.K.L. conceived and designed the study. R.S., E.C.O., I.M.G., C.O. J.N and M.K.L. performed the neutron scattering experiments and analyzed the data. R.S. E.C.O., I.M.G., and M.K.L. interpreted the data. R.S. and E.C.O. drafted the manuscript with input from and critical review by all authors. Based on their contribution, both R.S. and E.C.O. are considered as co-first author.

\section{COMPETING INTERESTS}

The authors declare no competing interests.

\section{ADDITIONAL INFORMATION}

Correspondence and requests for materials should be addressed to M.K.L.

Reprints and permission information is available at http://www.nature.com/ reprints

Publisher's note Springer Nature remains neutral with regard to jurisdictional claims in published maps and institutional affiliations.

Open Access This article is licensed under a Creative Commons Attribution 4.0 International License, which permits use, sharing, adaptation, distribution and reproduction in any medium or format, as long as you give appropriate credit to the original author(s) and the source, provide a link to the Creative Commons license, and indicate if changes were made. The images or other third party material in this article are included in the article's Creative Commons license, unless indicated otherwise in a credit line to the material. If material is not included in the article's Creative Commons license and your intended use is not permitted by statutory regulation or exceeds the permitted use, you will need to obtain permission directly from the copyright holder. To view a copy of this license, visit http://creativecommons. org/licenses/by/4.0/.

(c) The Author(s) 2021 